\title{
Variations of Lung Cancer Risk from Asbestos Exposure: Impact on Estimation of Population Attributable Fraction
}

\author{
Eun Kyeong MOON ${ }^{1}$, Mia SON ${ }^{2}$, Young-Woo JIN ${ }^{3}$, Sohee PARK ${ }^{4}$ and Won Jin LEE ${ }^{1 *}$ \\ ${ }^{1}$ Department of Preventive Medicine, College of Medicine, Korea University, Republic of Korea \\ ${ }^{2}$ Department of Preventive Medicine, College of Medicine, Kangwon National University, Republic of Korea \\ ${ }^{3}$ Department of Planning and Research for Radiological Emergency, National Rediation Emergency Medical \\ Center, Korea Institute of Radiological and Medical Science, Republic of Korea \\ ${ }^{4}$ Department of Epidemiology and Health Promotion, Yonsei University Graduate School of Public Health, \\ Republic of Korea
}

Received July 27, 2011 and accepted August 21, 2012

Published online in J-STAGE December 25, 2012

\begin{abstract}
The purpose of this study is to investigate the potential impact of differing lung cancer risks in study populations on estimating population attributable fraction (PAF) from asbestos exposure. Studies were identified via a MEDLINE search up to September 2009 and from the reference lists of publications about asbestos exposure and lung cancer risk. Relative risk estimates were extracted from 160 studies and meta-relative risks were calculated according to randomeffect models. Hypothetical PAFs were calculated based on the meta results and on the difference exposure scenarios. The risks for lung cancer from asbestos exposure were variable according to the region as well as other study characteristics. The risk estimates proved higher in Asian countries $(R R=3.53)$, in studies with 500 or fewer subjects $(R R=2.26)$, and papers published in the 1990s or earlier $(R R=1.91)$, than did those for European or North American countries, studies with more than 500 subjects, and papers published in the 2000s, respectively. The differences in PAFs between Asian and North American studies were 15.5\%, 30.3\%, and 36.2\% when the exposure prevalence was $10 \%, 30 \%$, and $50 \%$, respectively. This study suggested that it is important to apply appropriate lung cancer estimates to each study population when calculating PAF from asbestos exposure.
\end{abstract}

Key words: Asbestos exposure, Lung neoplasms, Meta-analysis, Population attributable fraction

\section{Introduction}

Population attributable fraction (PAF) is a useful index to quantify the burden of a given risk factor by combining relative risk with prevalence of exposure ${ }^{1)}$. Although PAF primarily depends on prevalence of exposure, another

*To whom correspondence should be addressed. Email: leewj@korea.ac.kr

(C)2013 National Institute of Occupational Safety and Health important determinant is the disease risk of the study population. However, it has been assumed that in many circumstances the relative risk (RR) remains essentially constant between populations when in fact it may vary considerably across different countries and time periods.

Asbestos ranks as one of the major occupational carcinogens and $5-7 \%$ of worldwide lung cancer cases may be attributable to occupational asbestos exposure ${ }^{2)}$. Previously, the most widely cited figures for calculating PAF from asbestos exposure are likely those published by 
Goodman $^{3)}$, which are based on meta-analysis of cohort studies, as in French ${ }^{4)}$ and $\mathrm{UK}^{5)}$ studies. World Health Organization used an estimate published by Steenland ${ }^{6}$ to assess the burden of lung cancer from occupational exposure $^{7)}$. However, it is difficult to be certain that a summary relative risk collected through meta-analysis is relevant to other populations due to likely differences in working conditions, exposure levels, and ethnic characteristics. Similarly, it is uncertain whether modern relative risks are relevant to past conditions and whether data from specific worksites are relevant to those in all other countries.

The aim of this study, therefore, is to produce updated estimates by different study characteristics of lung cancer risk due to asbestos exposure, and then to use these data to present potential differences for calculating PAF that will help to inform more appropriate future measures of lung cancer burden.

\section{Materials and Methods}

\section{Calculation of relative risk estimates}

The following medical subject headings and key words were used for searching out studies investigating the relation of asbestos exposure to lung cancer mortality or incidence: asbestos, lung, neoplasms, and risk. Original data for inclusion were identified through a MEDLINE search of literature published up to September 2009, without limitations on study period or language. In addition, the references in individual studies and reviews were similarly searched for further studies not obtained through the literature search. Through this process, a total of 7,394 studies were identified.

All titles and abstracts identified were screened and exclusion criteria were applied to the complete articles. Our exclusion criteria were: (a) not original research, not human study, (c) case reports and case series, (d) clinical lung cancer outcomes (e.g., diagnostic or therapeutic studies of lung cancer). Among the total, 7,088 studies were excluded and the remaining 306 studies were then reviewed in detail. By means of the detailed review of these articles, we also excluded (a) studies lacking an estimated risk outcome (e.g., RR, OR, SMR, SIR and 95\% CI) or sufficient data for calculation of the estimates, studies carried out on multiple exposures such as exposure to asbestos and other carcinogens, and (c) duplicated studies. When multiple studies had been published on an identical population, the studies with the longest follow-up period or the largest number of cases or the most recent publication date were selected. As a result, a total of 160 relevant
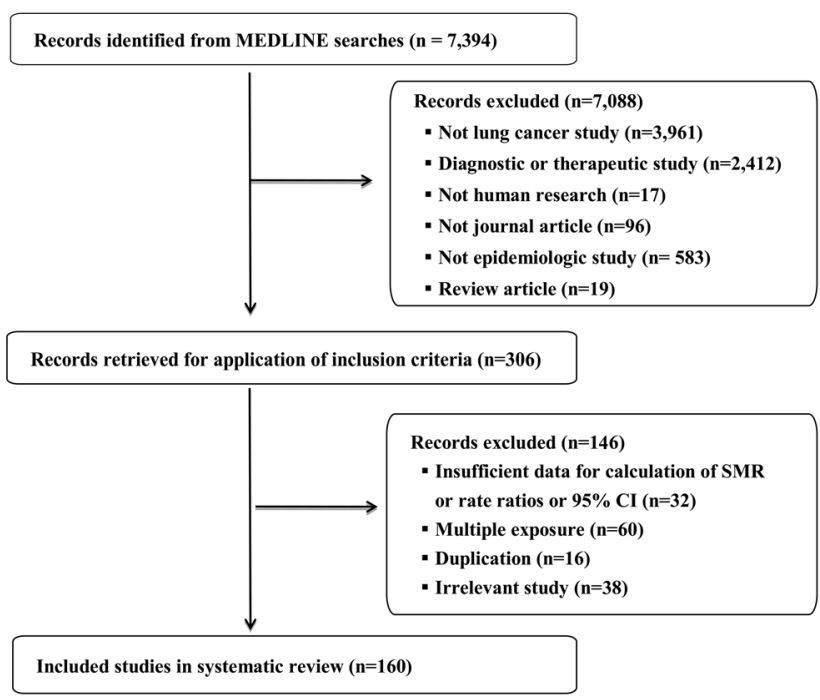

Fig. 1. Literature search process.

studies including 148 occupational and 12 environmental exposure studies were identified. A flowchart of the selection process is provided in Fig. 1.

Meta-analytic techniques that weight the logarithm of the RR of each study by a function of its variance were used to calculate a summary estimate. Due to a priori concerns regarding heterogeneity in the study populations, we used random-effects models for calculating summary statistics ${ }^{8)}$. Differing asbestos exposure levels were combined into ever exposure through meta-analysis of categories of individual studies. These analyses were performed both on the total data set and separately for study characteristics including country of study, design, size, year of publication, gender, and exposure type (i.e., occupational, environmental). All statistical computations were performed using the STATA software version 11.0 (Statacorp., College Station, TX).

\section{Calculation of hypothetical population attributable fraction}

The potential attributable fraction was calculated through the following equation ${ }^{9)}$ in which PAF denotes the population attributable fraction and where $\mathrm{P}(\mathrm{E})$ is the proportion of the population exposed to asbestos and RR is the relative risk a lung cancer arising an exposed individual compared with those who are unexposed.

$$
\mathrm{PAF}=\frac{P(E)(R R-1)}{1+P(E)(R R-1)}
$$

The meta-RRs from this study and hypothetical asbestos exposure prevalence ranging from $0 \%$ to $50 \%$ were ap- 
plied to estimate the PAF for lung cancer from asbestos exposure.

\section{Results}

The summary estimate from all studies showed a significant 1.8 fold-increased association for the relation between asbestos exposure and lung cancer (Table 1). The risk of lung cancer due to asbestos exposure showed wide variation. Furthermore, risk estimates were higher in Asian countries $(R R=3.53)$, in occupational exposure studies $(\mathrm{R}=1.88)$, in papers published in the 1990 s or earlier $(\mathrm{RR}=1.91)$, in studies with 500 or less subjects $(\mathrm{RR}=2.26)$ compared to those performed in Europe $(R R=1.63)$ and North America $(R R=1.49)$, in environmental exposure studies $(R R=1.06)$, in studies published in the 2000s $(R R=1.62)$, and in studies with 2000 more subjects $(R R=1.51)$, respectively. The majority of studies were occupational retrospective cohort studies $(n=112)$ and show relatively higher estimates than do case-control studies. The estimates did not meaningfully differ by gender and after adjusting for smoking. The European studies were conducted in the UK $(n=19)$, Italy $(n=19)$, Norway $(n=10)$, Sweden $(n=8)$, Finland $(n=7)$, Australia $(n=7)$, France $(n=5)$, and others while the North American studies took place in the US $(n=31)$ and Canada $(\mathrm{n}=5)$. Asian studies included China $(\mathrm{n}=23)$, Japan ( $\mathrm{n}=7)$, and Israel ( $\mathrm{n}=1)$ (data not shown).

Pooled results across regions are shown in Table 2. The results of the meta-analysis by study characteristics presented consistently higher risks in Asian studies than in others, and the estimates from North America and Europe were generally similar. The results from Asian studies on males showed especially high $R R s(R R=5.53$, 95\% CI 3.03-10.10) as did studies with 500 subjects or less ( $R R=5.48,95 \%$ CI 3.44-8.73). However, the number of Asian studies was relatively small compared to those performed in Western countries.

The hypothetical PAFs were calculated using a range of scenarios of asbestos exposure prevalence and metaRRs from this study (Fig. 2). The considerable variability in PAF estimates is mainly because of the high degree of variability by country. The PAFs for lung cancer grew with increasing exposure prevalence and the highest PAFs were found among Asian populations. The difference in PAFs between Asians and North Americans were 15.5\%, 30.3\%, and $36.2 \%$ when the exposure prevalence was $10 \%, 30 \%$, and $50 \%$, respectively. PAFs for lung cancer from asbestos exposure were also varied by exposure types, publication years, and population size (data not shown).
Table 1. Summary RRs for the relation between lung cancer and asbestos exposure

\begin{tabular}{|c|c|c|c|}
\hline Categories & No. of studies & Meta- RR & $95 \% \mathrm{CI}$ \\
\hline All studies & 160 & 1.81 & $1.68-1.95$ \\
\hline \multicolumn{4}{|l|}{ Region } \\
\hline Asia & 31 & 3.53 & $2.59-4.79$ \\
\hline US \& Canada & 36 & 1.49 & $1.25-1.77$ \\
\hline Europe & 92 & 1.63 & $1.49-1.77$ \\
\hline Africa & 1 & 1.72 & $1.33-2.23$ \\
\hline \multicolumn{4}{|l|}{ Study design } \\
\hline Cohort & 115 & 1.84 & $1.68-2.02$ \\
\hline Retrospective & 112 & 1.87 & $1.70-2.05$ \\
\hline Prospective & 3 & 1.3 & $1.15-1.46$ \\
\hline Case-control & 45 & 1.74 & $1.49-2.03$ \\
\hline Hospital-based & 17 & 1.75 & $1.44-2.12$ \\
\hline Population-based & 28 & 1.7 & $1.37-2.10$ \\
\hline \multicolumn{4}{|l|}{ Exposure type } \\
\hline Occupational & 148 & 1.88 & $1.74-2.04$ \\
\hline Environmental & 12 & 1.06 & $0.86-1.30$ \\
\hline \multicolumn{4}{|l|}{ Gender } \\
\hline Male & 69 & 1.71 & $1.51-1.94$ \\
\hline Female & 20 & 1.99 & $1.46-2.71$ \\
\hline Mixed & 71 & 1.88 & $1.68-2.11$ \\
\hline \multicolumn{4}{|l|}{ Published year } \\
\hline$\leq 1990 \mathrm{~s}$ & 117 & 1.91 & $1.72-2.11$ \\
\hline$\geq 2000 \mathrm{~s}$ & 43 & 1.62 & $1.42-1.85$ \\
\hline \multicolumn{4}{|l|}{ Smoking adjustment } \\
\hline Yes & 38 & 1.7 & $1.48-1.97$ \\
\hline No & 122 & 1.85 & $1.68-2.04$ \\
\hline \multicolumn{4}{|l|}{ Population size } \\
\hline$\leq 500$ & 56 & 2.26 & $1.87-2.72$ \\
\hline $501 \sim 1,000$ & 27 & 2.03 & $1.60-2.56$ \\
\hline $1001 \sim 2,000$ & 21 & 1.6 & $1.27-2.01$ \\
\hline$\geq 2,001$ & 56 & 1.51 & $1.37-1.66$ \\
\hline
\end{tabular}

\section{Discussion}

Our findings demonstrated a potential difference in PAFs with different estimates on lung cancer and asbestos exposure. The biggest difference was observed in geographical area; therefore, the use of appropriate regionspecific risk estimates is necessary to obtain more exact PAFs. To our knowledge, this is the first study to evaluate the potential impact on the calculation of PAF for lung cancer associated with asbestos exposure using estimates of RRs derived through meta-analysis.

The extensive regional difference in lung cancer estimates implies that exposure to lung carcinogens varies greatly between nations. Possible reasons for the elevated 
Table 2. Summary RRs for the relation between lung cancer and asbestos exposure by region

\begin{tabular}{|c|c|c|c|c|c|c|c|c|c|}
\hline & \multicolumn{3}{|c|}{ Asia } & \multicolumn{3}{|c|}{ US \& Canada } & \multicolumn{3}{|c|}{ Europe } \\
\hline & No. of studies & Meta-RR & $95 \% \mathrm{CI}$ & No. of studies & Meta-RR & $95 \% \mathrm{CI}$ & No. of studies & Meta-RR & $95 \% \mathrm{CI}$ \\
\hline \multicolumn{10}{|l|}{ Exposure type } \\
\hline Occupational & 30 & 3.65 & $2.66-5.00$ & 31 & 1.62 & $1.35-1.95$ & 86 & 1.65 & $1.51-1.81$ \\
\hline Environmental & 1 & 1.43 & $0.89-2.29$ & 5 & 0.78 & $0.47-1.26$ & 6 & 1.15 & $0.94-1.40$ \\
\hline \multicolumn{10}{|l|}{ Study design } \\
\hline Cohort & 31 & 3.53 & $2.59-4.79$ & 24 & 1.38 & $1.13-1.68$ & 59 & 1.60 & $1.44-1.77$ \\
\hline Case-control & - & - & - & 12 & 1.75 & $1.27-2.41$ & 33 & 1.67 & $1.46-1.92$ \\
\hline \multicolumn{10}{|l|}{ Gender } \\
\hline Male & 7 & 5.53 & $3.03-10.10$ & 15 & 1.35 & $1.16-1.58$ & 42 & 1.65 & $1.43-1.90$ \\
\hline Female & 6 & 4.00 & $3.30-4.83$ & 2 & 1.34 & $0.95-1.90$ & 7 & 1.88 & $1.39-2.54$ \\
\hline Mixed & 17 & 2.93 & $2.08-4.12$ & 14 & 1.96 & $1.50-2.58$ & 37 & 1.63 & $1.43-1.85$ \\
\hline \multicolumn{10}{|l|}{ Published year } \\
\hline$\leq 1990$ s & 24 & 3.79 & $2.59-5.56$ & 30 & 1.57 & $1.29-1.92$ & 62 & 1.66 & $1.50-1.85$ \\
\hline$\geq 2000$ & 7 & 2.81 & $1.54-5.13$ & 6 & 1.18 & $0.85-1.63$ & 30 & 1.56 & $1.34-1.81$ \\
\hline \multicolumn{10}{|c|}{ Smoking adjustment } \\
\hline Yes & 3 & 2.06 & $1.05-4.03$ & 8 & 1.93 & $1.32-2.80$ & 24 & 1.61 & $1.38-1.88$ \\
\hline No & 27 & 3.85 & $2.77-5.35$ & 23 & 1.53 & $1.27-1.85$ & 62 & 1.66 & $1.48-1.87$ \\
\hline \multicolumn{10}{|l|}{ Population size } \\
\hline$\leq 500$ & 13 & 5.48 & $3.44-8.73$ & 9 & 1.69 & $1.32-2.15$ & 34 & 1.78 & $1.49-2.12$ \\
\hline $501-1,000$ & 8 & 3.27 & $2.33-4.60$ & 8 & 1.73 & $1.08-2.78$ & 11 & 1.72 & $1.31-2.27$ \\
\hline $1001-2,000$ & 3 & 3.31 & $1.18-9.33$ & 7 & 1.00 & $0.58-1.70$ & 11 & 1.71 & $1.23-2.37$ \\
\hline$\geq 2,001$ & 7 & 2.10 & $1.19-3.68$ & 12 & 1.43 & $1.20-1.71$ & 36 & 1.43 & $1.27-1.61$ \\
\hline
\end{tabular}

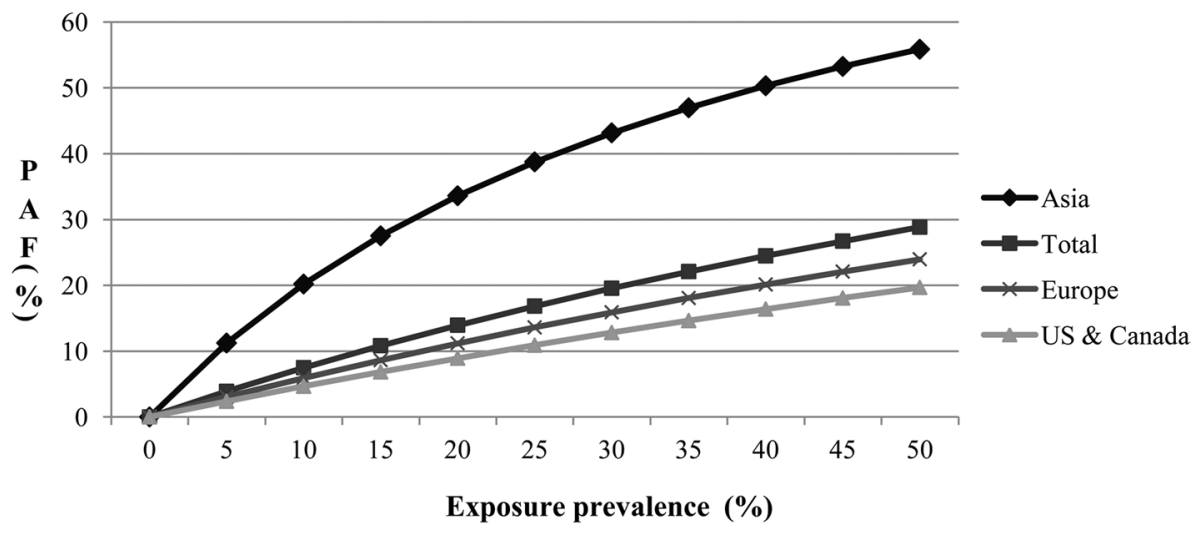

Fig. 2. Estimates of the population attributable fraction of lung cancer associated with asbestos exposure by region.

estimates in Asia include that exposure to asbestos may be higher in Asia than on other continents. Although the use of asbestos has been banned or subject to strict control in most Western countries, some asbestos products are still used in Eastern Europe, Latin America, and Asia ${ }^{10)}$. Asbestos consumption in Asia increased from an estimated $977,599 \mathrm{ton} / \mathrm{yr}$ in 1990 to $1,261,128$ in 1995. On the other hand, Unite States and Canadian consumption declined from an estimated 150,958 ton/yr to 53,158 over the same period. That in Europe similarly fell from an estimated 2,582,294 ton/yr to 926,994 although asbestos had more consumed in Europe than other regions before 1990 ${ }^{11)}$. In addition, the types and sizes of asbestos exposure may different by countries. Differences in exposure between countries may arise from divergent legislation, technology, culture and attitude toward risk. 
A synergistic effect between tobacco smoke and asbestos in the causation of lung cancer ${ }^{12,13)}$ may also explain this result. The prevalence of male smokers has generally decreased in Europe during the last decade, whereas the smoking rate remains high in many Asian countries ${ }^{14)}$. Therefore, the risk of lung cancer due to asbestos exposure may increase in Asian populations due to a high smoking prevalence, even though exposure levels may be similar with other countries.

Biological differences may also influence the relationship between asbestos exposure and lung cancer by population. Previous studies have shown that polymorphisms in glutathione S-transferase isoenzymes and microsomal epoxide hydrolase may affect the risk for asbestos-related diseases ${ }^{15,16)}$. PON1 polymorphism may also be an important risk factor for chronic diseases, including lung cancer, and is known to have a pronounced variability across different ethnic groups ${ }^{17)}$. A combination of differences from exposure, smoking prevalence, and genotype may produce the wide variation in RRs and corresponding PAFs in this study. Therefore, relative risk measured in one country under particular circumstances or a simple summary RR may not be applicable to a specific population in a different country.

We also found different relative risks for lung cancer by publication year of studies, which may correlate with years of exposure to asbestos. Asbestos exposure may vary over time due to changing usage, control measures, and working practices and has probably decreased over time due to general improvements in technology ${ }^{18)}$. Our results showed that the lung cancer risks in studies published in the 1990s or earlier were higher than those from the 2000s or later in all countries; therefore, differences in exposure over time are also of interest in assessing the occupational PAFs for lung cancer even within the same country.

No significant association was observed between lung cancer and environmental asbestos exposure, whereas occupational exposure showed a significantly increased risk. The levels of environmental exposure to asbestos are generally low, and the duration and frequency of exposure are seldom precisely discernable with environmental exposure $^{19,20)}$. Therefore, a high degree of heterogeneity may exist between studies of environmental exposure to asbestos.

The reason for relatively high RRs in few-subject studies may relate to different exposure levels. Relatively smaller-scale industries may have higher exposure due to poor working conditions. Therefore the lung cancer risk in small studies can be higher than in larger studies. Unstable results due to small sample size offer another possible explanation since smaller studies tend more to induce bias away from null than do larger studies ${ }^{21)}$.

Other differences in study design, asbestos types, subject selection, exposure patterns, diagnostic criteria, potential confounding factors and statistical techniques may explain the observed heterogeneity of risk estimates. Additionally, occupational cohort studies may vary greatly by industry, asbestos fiber type, and the demographic characteristics of cohort members ${ }^{3)}$.

There are certain important limitations to this study. First, considerable heterogeneity may be present in the exposure assessment methods in the studies. Considering that the majority of studies are based on job exposure matrix or expert assessment, exposure information in this study may be inconsistent and there may be a degree of non-differential exposure misclassification. Second, we did not assess the methodological quality of the studies and hence did not exclude studies on the basis of quality score. Instead, we performed subgroup analyses according to study feature that could potentially affect the heterogeneity of individual studies.

Despite such limitations, we have conducted metaanalysis to evaluate the most up to date available RRs between lung cancer and asbestos exposure. These results demonstrated that the risks for lung cancer from asbestos exposure are variable according to the area under study, exposure type, population size, and year of publication. Our study differs from earlier reviews in that we have applied meta-results to the calculation of PAFs for lung cancer from asbestos exposure. Asian studies had the highest risk estimates and correspondingly produced the highest PAF. PAFs for lung cancer from asbestos exposure varied with different risk estimates at the same exposure prevalence. Therefore, caution is required using a single estimate for PAF, and it is important to apply adequate lung cancer estimates for each study population when calculating PAF to estimate the most appropriate disease burden in a country.

\section{Acknowledgement}

This study was supported by Korea University Grant (K1031681) and by a grant from the National R\&D program for Cancer Control, Ministry for Health and Welfare, Republic of Korea (0820280). 


\section{Competing Interests}

None.

\section{Ethics Statement}

This study analyzed publicly available data sets and was therefore exempt from institutional review board approval.

\section{References}

1) Steenland K, Armstrong B (2006) An overview of methods for calculating the burden of disease due to specific risk factors. Epidemiology 17, 512-9. [Medline] [CrossRef]

2) LaDou J (2004) The asbestos cancer epidemic. Environ Health Perspect 112, 285-90. [Medline] [CrossRef]

3) Goodman M, Morgan RW, Ray R, Malloy CD, Zhao K (1999) Cancer in asbestos-exposed occupational cohorts: a meta-analysis. Cancer Causes Control 10, 453-65. [Medline] [CrossRef]

4) Boffetta $\mathrm{P}$, Autier $\mathrm{P}$, Boniol M, Boyle $\mathrm{P}$, Hill C, Aurengo A, Masse R, The G, Valleron AJ, Monier R, Tubiana M (2010) An estimate of cancers attributable to occupational exposures in france. J Occup Environ Med 52, 399-406. [Medline] [CrossRef]

5) Rushton L, Bagga S, Bevan R, Brown TP, Cherrie JW, Holmes P, Fortunato L, Slack R, Van Tongeren M, Young C, Hutchings SJ (2010) Occupation and cancer in Britain. Br J Cancer 102, 1428-37. [Medline] [CrossRef]

6) Steenland K, Loomis D, Shy C, Simonsen N (1996) Review of occupational lung carcinognes. Am J Ind Med 29, 474-90. [Medline] [CrossRef]

7) World Health Organization (2004) Occupational carinogens: assessing the environmental burden of disease at national and local levels. WHO, Geneva.

8) DerSimonian R, Laird N (1986) Meta-analysis in clinical trials. Control Clin Trials 7, 177-88. [Medline] [CrossRef]

9) Levin ML (1953) The occurrence of lung cancer in man. Acta Unio Int Contra Cancrum 9, 531-41. [Medline]

10) Lotti M, Bergamo L, Murer B (2010) Occupational toxicology of asbestos-related malignancies. Clin Toxicol (Phila) 48, 485-96. [Medline] [CrossRef]
11) Virta RL (2006) Worldwide Asbestos Supply and Consumption Trends from 1900 through 2003. U.S. Geological Survey, Reston.

12) Wraith D, Mengersen K (2008) A Bayesian approach to assess interaction between known risk factors: the risk of lung cancer from exposure to asbestos and smoking. Stat Methods Med Res 17, 171-89. [Medline] [CrossRef]

13) Frost G, Darnton A, Harding AH (2011) The effect of smoking on the risk of lung cancer mortality for asbestos workers in Great Britain (1971-2005). Ann Occup Hyg 55, 239-47. [Medline] [CrossRef]

14) Yoo KY (2010) Cancer prevention in the Asia pacific region. Asian Pac J Cancer Prev 11, 839-44. [Medline]

15) Neri M, Taioli E, Filiberti R, Paolo Ivaldi G, Aldo Canessa $P$, Verna A, Marroni P, Puntoni R, Hirvonen A, Garte S (2006) Metabolic genotypes as modulators of asbestos-related pleural malignant mesothelioma risk: a comparison of Finnish and Italian populations. Int J Hyg Environ Health 209, 393-8. [Medline] [CrossRef]

16) Hirvonen A, Pelin K, Tammilehto L, Karjalainen A, Mattson K, Linnainmaa K (1995) Inherited GSTM1 and NAT2 defects as concurrent risk modifiers in asbestosrelated human malignant mesothelioma. Cancer Res 55, 2981-3. [Medline]

17) Mohamed Ali S, Chia SE (2008) Interethnic variability of plasma paraoxonase (PON1) activity towards organophosphates and PON1 polymorphisms among Asian populations-a short review. Ind Health 46, 309-17. [Medline] [CrossRef]

18) Cherrie JW, Van Tongeren M, Semple S (2007) Exposure to occupational carcinogens in great britain. Ann Occup Hyg 51, 653-64. [Medline] [CrossRef]

19) Boffetta $P$ (2004) Epidemiology of environmental and occupational cancer. Oncogene 23, 6392-403. [Medline] [CrossRef]

20) Bourdès V, Boffetta P, Pisani P (2000) Environmental exposure to asbestos and risk of pleural mesothelioma: review and meta-analysis. Eur J Epidemiol 16, 411-7. [Medline] [CrossRef]

21) Nemes S, Jonasson JM, Genell A, Steineck G (2009) Bias in odds ratios by logistic regression modelling and sample size. BMC Med Res Methodol 9, 56. [Medline] [CrossRef] 